
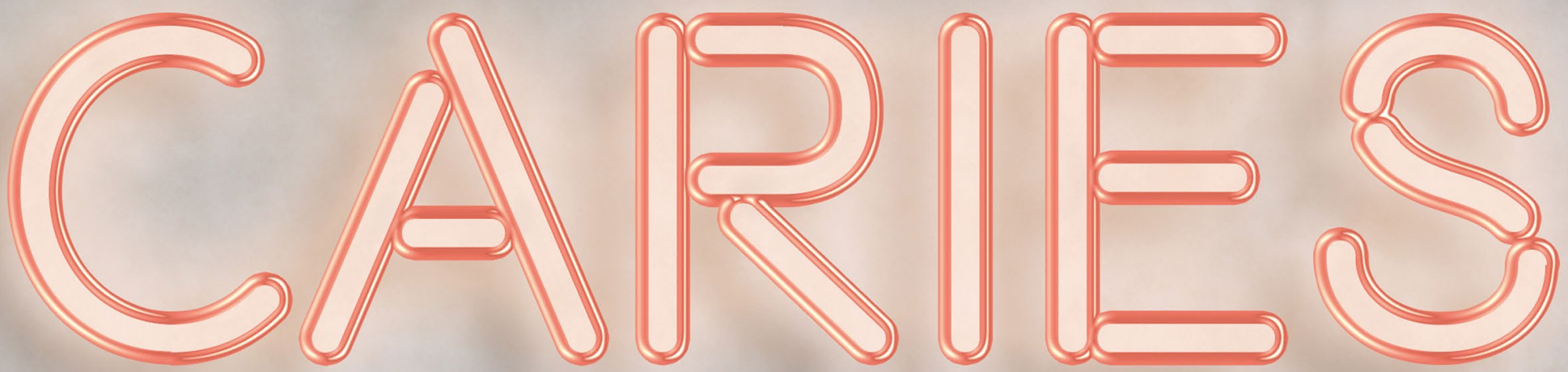

\title{
Shining a light on caries: evaluating the evidence for diagnostic test accuracy of different illumination tests for the detection and diagnosis of enamel caries
}

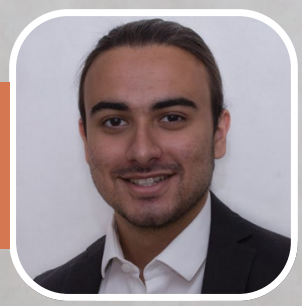

\author{
By Joshua J. S. Hurley, a dental \\ student at King's College London
}

\section{Abstract}

Dental caries is a highly prevalent disease which dental practitioners face daily. One of the key aspects of managing this disease when prevention has failed is diagnosis and being able to spot the caries; traditional methods include visual examination and probing for soft tissue. Three modern technologies: optical coherence tomography (OCT), fibre-optic transillumination (FOTI) and near-infrared transillumination (NIR), have been developed and sold by manufacturers to practitioners to aid the process of caries detection, but just how good are these technologies and are they accurate enough to be used in clinical practice?

This article summarises the latest research on each of the methods, determines the positives and negatives of each instrument and determines the ability for them to detect caries.

The key findings were that NIR has a similar ability to detect caries to bitewing $\mathrm{X}$-rays and could be considered as an alternative to radiographs; FOTI is better for elimination of healthy teeth rather than detecting teeth with caries; and OCT is most sensitive but is not readily available for general practice and can have problems with penetration depth. In summary, all three technologies should be used to supplement traditional methods and not be stand-alone methods of caries detection. The most accessible and useful device currently seems to be NIR but all the devices have different applications outside of caries detection which need to be researched further so that clinicians can best choose which devices they want to use and for which applications. 


\section{Introduction}

Dental caries is the most common non-communicable disease across the world according to the World Health Organisation. ${ }^{1}$ Caries leading to cavities is both preventable and reversible if caught at the onset. To identify caries most general dental practitioners (GDPs) probe the teeth to check for soft tissue (students are now taught to use a 'gentle' probe) and use visual examinations. ${ }^{2}$ An alternative to these traditional methods is using radiographs; however, for the safety of the patient it is best to minimise exposure to ionising radiation as much as possible. It is important for early detection of caries to allow potential remineralisation of previously carious tissue. If caries is detected late, after the opportunity for remineralisation, the main option is a filling to fix the damage.

Three modern technologies (optical coherence tomography, fibre-optic transillumination and near-infrared transillumination) offer ways for clinicians to identify caries without using radiation, but just how good are these techniques? The objective of this article is to compile the latest research on illumination tests in comparison to traditional methods of caries detection so that their feasibility and usefulness can be deduced.

\section{Optical coherence tomography}

Optical coherence tomography (OCT) uses a complex of mirrors and half mirrors, as shown in Figure 1, to build a reflection pattern which ultimately leads to a cross section.

Tomography is a cross sectional representation of a solid sample (usually biological tissue). Devices are thought to have 'superior sensitivity to NIR and fibre-optic devices' ${ }^{3}$ however they are limited and not developed enough for use in general practices. The benefits and negatives are summarised by Yao-Sheng Hsieh et al. ${ }^{4}$ where they found the benefits of OCT were: a high spatial resolution, real-time imaging and a possibility of $3 \mathrm{D}$ reconstruction. The main negative stated was a 'limited penetration depth and scanning range.. ${ }^{4}$ Penetration depth was also presented as a limitation by $\mathrm{A}$. Baumgartner et al. ${ }^{5}$ This is due to the 'light attenuation from scattering by tissue, ; another limitation identified by this paper is that there is a significant influence of the properties of biological tissues, such as the refractive index.

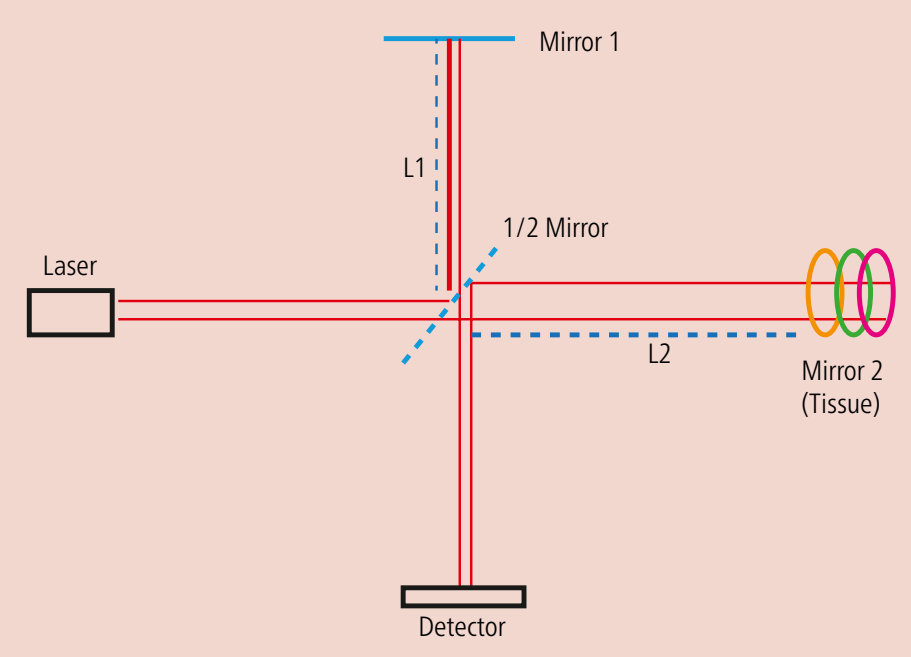

Fig. 1 OCT mirror complex

\begin{tabular}{|c|c|c|}
\hline $\begin{array}{l}\text { Occlusal and approximal } \\
\text { surfaces }\end{array}$ & Schneiderman and others ${ }^{25}$ & $\begin{array}{l}\text { Sensitivity } 0.67 \text { for occlusal } \\
\text { surfaces and } 0.56 \text { for } \\
\text { approximal surfaces; } \\
\text { radiography had sensitivity of } \\
0.18 \text { and } 0.21 \text { respectively }\end{array}$ \\
\hline In vitro use & Peers and others ${ }^{26}$ & Sensitivity 0.67 , specificity 0.97 \\
\hline Occlusal caries & Mitropoulos ${ }^{27}$ & Sensitivity 0.85 , specificity 1.00 \\
\hline Buccal surfaces & Sidi and Naylor ${ }^{28}$ & Sensitivity 0.74 , specificity 0.99 \\
\hline
\end{tabular}

${ }^{6}$ It is important for early detection of caries to allow potential remineralisation

of previously carious tissue. If caries is detected late, after the opportunity

\section{for remineralisation, the main option}

\section{is a filling to fix the damage.}

Monika Machoy et al. ' states 'OCT allows for soft-tissue imaging, which is important in the treatment of periodontal diseases' when discussing OCT in practice. However, around caries detection it recognises the unreliability of the technology but does conclude that 'the possibility of early diagnosis of caries [...] has already been proven'?
It is important to recognise that there are different types of OCT including Polarisation Sensitive OCT (PS-OCT). 'PS-OCT performs rapid two- and three-dimensional imaging of transparent and translucent samples with micrometre scale resolution. ${ }^{8}$ PS-OCT seems to have advantages over OCT as it 'reduces banding artifacts and provides additional 
functionality through phase retardation information.' OCT can also vary based on the lenses used and make and model of the technology.

\section{Fibre-Optic Transillumination}

Fibre-Optic Transillumination (FOTI) uses the idea of total internal reflection, when light travels from a high to low index of refraction at an angle greater than the critical angle. This reflects light along the cable until reaching a small aperture where light is emitted. It is important to note there are multiple types of FOTI including DIFOTI (Digital Imaging FOTI) which may be included in studies. said to be most useful in a dentition with no or few fillings - using FOTI in younger dentitions is when it presents as most useful. ${ }^{14}$ When compared to clinical visual examination and bitewing radiography, FOTI 'detected more initial and manifested caries lesions.' ${ }^{14}$

DIFOTI allows images to be captured and stored digitally and increases convenience for GDPs. Iain A. Pretty and Gerardo Maupomé conclude: 'DIAGNODent [laser device], DIFOTI and QLF [quantitative light-induced fluorescence] devices may improve decision making by affording more sophisticated diagnostic and management capabilities

\section{"FOTI seems to be regarded as a useful} and accurate tool due to the

\section{small aperture of light resulting in high} focus, however it is still recommended

\section{to be used supplementally.}

Table 1 shows the sensitivity (person with caries shown to have caries) and the specificity (person without caries shown to not have caries) of FOTI devices. The data in Cariology ${ }^{10}$ concluded that the specificity is higher than the sensitivity of FOTI, so FOTI may be more useful for correct diagnosis if exclusion is used (identify those without caries instead of identifying those with caries and use elimination). This is supported by Richard Macey et al.: ${ }^{3}$ 'limitations in their [NIR and FOTI] ability to detect enamel caries'; in contrast it states that FOTI devices are good at identifying healthy teeth. Cariology ${ }^{10}$ also shows limitations: 'FOTI can only be used for coronal tooth surfaces (occlusal, interproximal, and smooth) and not below the gingiva.' ${ }^{11}$

FOTI seems to be regarded as a useful and accurate tool due to the small aperture of light resulting in high focus, however it is still recommended to be used supplementally. ${ }^{13}$ FOTI may improve treatment procedures in alternative ways such as: 'detection of calculus, evaluation of soft-tissue lesions, used to rule out fractures in ceramic restorations before cementation and other clinical features. ${ }^{13}$ FOTI is also (through more detailed information) and by providing a clearly stated measure of longitudinal lesion activity'. ${ }^{12}$ As previously mentioned, it is important to note that most studies on FOTI recognise it as supplementary technology but not the primary method for caries detection.

\section{Near-Infrared}

Near-Infrared (NIR) technology uses wavelengths in the range of 780-1550 nm, slightly longer than red visible light. A charge coupled device (a highly sensitive photon detector) is used to translate the detected NIR light into an image.

A. M. Maia et al. ${ }^{15}$ compared NIR to dental radiographs for caries detection; results show that NIR sensitivity and specificity provide good results in comparison to radiographs. The study concludes that NIR 'can improve routine monitoring of enamel lesions. ${ }^{15}$ It also acknowledges that NIR has good 'potential for imaging early approximal enamel lesions ${ }^{15}$ to aid preventative intervention. Ismail Hakki Baltacioglu and Kaan Orhan ${ }^{16}$ support the idea of NIR being a valid alternative to radiography and explain the biggest advantage in comparison to X-rays is that NIR is non-ionising radiation.

The in vivo study by Jan Kuhnisch et al. ${ }^{17}$ compares NIR to bitewing radiographs and notes that NIR 'achieved the same level as bitewing ${ }^{17}$ for proximal dentine caries detection. The identification of dentine caries was supported by Friederike Litzenburger et al..$^{18} \mathrm{~A}$ similar study notes 'reasonably comparable accuracy to that of bitewing for detecting interproximal carious lesions. ${ }^{19}$ An in vivo study by Cynthia LaraCapi et al. ${ }^{20}$ concluded: 'Transillumination (NIR) showed to be a reliable method and as effective as traditional methods in caries detection (bitewings and clinical examination)'.

A big advantage noted by Keith Angelino et al. ${ }^{21}$ was that the NIR system could display subsurface caries. This includes 'those around fillings, demineralisation, fractures, and cracks. ${ }^{21}$

NIR systems can vary; Yihua Zhu et al. ${ }^{22}$ conducted a study on a Dual Short Wavelength Infrared Probe. Results showed that having a transillumination mode (for approximal lesions) and a reflectance mode (for occlusal lesions) complemented each other; 'for both types of lesions and possible false positives appearing in reflectance mode can be verified in transillumination mode..22 This presents a strong case for the use of dual wavelength devices.

\section{Discussion}

Out of the three technologies discussed, the least likely to be used in general practice is OCT as the systems are not available. It does however have alternative uses on top of caries detection; much like FOTI, devices can be used for observing soft tissues along with hard tissues. FOTI doesn't seem to be able to detect enamel caries as well as OCT, however when used for exclusion of healthy teeth good results were shown (this is not an efficient way of detecting caries but has a place in general dentistry). FOTI is compared to NIR which is said to be like bitewing radiographs. NIR is recognised as a possible alternative to radiographs; this is supported by the fact that NIR can image around fillings and fractures in teeth.

All the technologies have variants with their own benefits and setbacks. All three technologies have a digital variant allowing clear examination for the practitioner; FOTI can also be used non-digitally as a basic light to illuminate caries. It is also important to recognise that all these devices 
can have alternative uses to caries detection, such as FOTI being used for examining soft tissue lesions. and diagnosis of enamel caries. Cochrane Database Syst Rev 2021; CD013855.

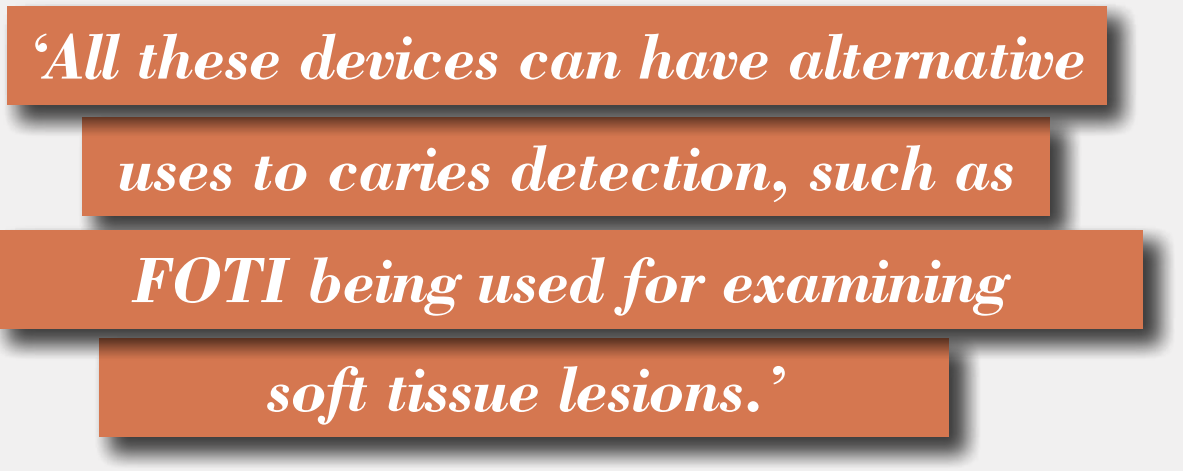

To summarise: OCT has greater sensitivity to NIR and FOTI, however, has poor penetration depth, is highly affected by tissue refractive indexes and is not readily available for general practice. FOTI is limited in its ability to detect enamel caries but is useful and accurate at identifying healthy teeth (caries detection by exclusion); it is only recommended for FOTI to be used supplementally. NIR has a similar sensitivity and specificity to bitewing radiographs and is thought to be good at detecting early enamel lesions. NIR can also achieve the same results as bitewing radiographs for dentine caries detection and is able to display subsurface caries. Further research into the other functions would be useful so that practitioners could select the device that best suits their needs.

\section{Acknowledgements}

Anitha Mclean, Deiko Iverieli, Eithar Ibrahim, Fionnuala Pye, Mehr Nickfarjam This article is based on a King's College London Faculty of Dentistry, Oral \& Craniofacial Sciences Student Selected Component Poster Presentation.

\section{References}

1. World Health Organization. Sugars and dental caries. 9 November 2017. Available at: https://www.who.int/news-room/factsheets/detail/sugars-and-dental-caries (accessed 21 May 2021).

2. Mayo Clinic. Cavities/tooth decay. 2017. Available at: https://www.mayoclinic.org/ diseases-conditions/cavities/diagnosistreatment/drc-20352898 (accessed 21 May 2021).

3. Macey R, Walsh T, Riley P et al. Transillumination and optical coherence tomography for the detection
4. Hsieh Y S, Ho Y C, Lee S Y et al. Dental optical coherence tomography. Sensors (Basel) 2013; 13: 8928-8949.

5. Baumgartner A, Dichtl S, Hitzenberger $\mathrm{C} \mathrm{K}$ et al. Polarization-sensitive optical coherence tomography of dental structures. Caries Res 2000; 34: 59-69.

6. Shimada Y, Tagami J, Sumi Y. (2014). Potential and limitations of OCT for assessing dental caries and tooth crack. J Japanese Soc Laser Dent 2014; 25: 159-164.

7. Machoy M, Seeliger J, SzyszkaSommerfeld L, Koprowski R, Gedrange T, Woźniak, K. (2017). The use of optical coherence tomography in dental diagnostics: a state-of-theart review. J Healthc Eng 2017; doi: 10.1155/2017/7560645.

8. Baumann B. Polarization Sensitive Optical Coherence Tomography: A Review of Technology and Applications. Appl Sci 2017; doi: 10.3390/app7050474.

9. Sahyoun C C, Subhash H M, Peru D, Ellwood R P, Pierce M C. An experimental review of optical coherence tomography systems for noninvasive assessment of hard dental tissues. Caries Res 2020; 54: 43-54.

10.Wikifoundry. Cariology. 2010. Available at: http://cariology.wikifoundry.com/ page/Fiber+Optic+Transillumination (accessed 21 May 2021).

11.Pretty I A. Caries detection and diagnosis: novel technologies. J Dent 2006; 34: 727-739.

12.Pretty I, Maupomé G. A closer look at diagnosis in clinical dental practice: Part 5. Emerging technologies for caries detection and diagnosis. J Can Dent Assoc 2004; 70: 540, 540a-540i.

13.Strassler H E, Pitel M L. Using fiber-optic transillumination as a diagnostic aid in dental practice. Compend Contin Educ Dent 2014; 35: 80-88.

14.Laitala M-L, Piipari L, Sämpi N et al. Validity of digital imaging of fiber-optic transillumination in caries detection on proximal tooth surfaces. Int J Dent 2017; doi: $10.1155 / 2017 / 8289636$.

15. Maia A M A, Karlsson L, Margulis W, Gomes A S L. Evaluation of two imaging techniques: near-infrared transillumination and dental radiographs for the detection of early approximal enamel caries. Dentomaxillofac Radiol 2011; 40: 429-433.

16. Baltacioglu I H, Orhan, K. Comparison of diagnostic methods for early interproximal caries detection with near-infrared light transillumination: an in vivo study. BMC Oral Health 2017; 17: 130. doi: 10.1186/s12903-017-0421-2.

17. Kühnisch J, Söchtig F, Pitchika V et al. In vivo validation of near-infrared light transillumination for interproximal dentin caries detection. Clin Oral Investig 2016; 20: 821-829.

18.Litzenburger F, Schäfer G, Hickel R, Kühnisch J, Heck K. Comparison of novel and established caries diagnostic methods: a clinical study on occlusal surfaces. BMC Oral Health 2021; 21: doi: 10.1186/s12903-021-01465-8.

19. Ortiz M I G, de Melo Alencar C, De Paula B L F, Magno M B, Maia L C, Silva C M. Accuracy of near-infrared light transillumination (NILT) compared to bitewing radiograph for detection of interproximal caries in the permanent dentition: A systematic review and metaanalysis. J Dent 2020; 98: doi: 10.1016/j. jdent.2020.103351.

20.Lara-Capi C, Cagetti M G, Lingström $\mathrm{P}$ et al. Digital transillumination in caries detection versus radiographic and clinical methods: an in-vivo study. Dentomaxillofac Radiol 2017; 46: doi: 10.1259/dmfr.20160417.

21. Angelino K, Edlund D A, Shah P. Nearinfrared imaging for detecting caries and structural deformities in teeth. IEEE $J$ Transl Eng Health Med 2017; 5: doi: 10.1109/JTEHM.2017.2695194.

22.Zhu Y, Chang N Y, Fried W A, Yang V, Fried D. A dual handheld SWIR transillumination/reflectance probe for imaging lesions on tooth occlusal and proximal surfaces. Proc SPIE Int Soc Opt Eng 2020; doi: 10.1117/12.2550984.

https://doi.org/10.1038/s41407-021-0716-7 